\title{
La lettre de doléance dans la zone française d'occupation entre 1945 et 1949
}

Gesuchs- und Beschwerdebriefe zwischen 1945 und 1949

The Complain and Request Letter between 1945 and 1949

\section{Marjorie Maquet}

\section{(2) OpenEdition}

Journals

Édition électronique

URL : http://journals.openedition.org/ceg/742

DOI : $10.4000 /$ ceg. 742

ISSN : 2605-8359

Éditeur

Presses Universitaires de Provence

Édition imprimée

Date de publication : 18 novembre 2016

Pagination : 209-219

ISBN : 979-10-320-0087-8

ISSN : 0751-4239

\section{Référence électronique}

Marjorie Maquet, «La lettre de doléance dans la zone française d'occupation entre 1945 et 1949 »,

Cahiers d'Études Germaniques [En ligne], 71 | 2016, mis en ligne le 18 mai 2018, consulté le 10

décembre 2020. URL : http://journals.openedition.org/ceg/742 ; DOI : https://doi.org/10.4000/ceg.742

Tous droits réservés 


\title{
La lettre de doléance dans la zone française d'occupation entre 1945 et 1949
}

\author{
Marjorie MAQUET \\ Université Toulouse Jean-Jaurès
}

Il est établi que l'intérêt de la recherche envers la production épistolaire dépasse son apport biographique ou historique. Les Actes d'un colloque qui s'est déroulé à Calais et qui avait été consacré à l'étude des liens entre politique et épistolaire montrent que ce sont " moins des faits historiques qui sont recherchés (bien que cet apport ne soit pas à négliger) que leur perception, moins des théories politiques que des lieux où elles s'élaborent et se diffusent ${ }^{1}$. " Outre sur la relation entre l'épistolaire et le politique, largement étudiée entre autres lors de ce colloque, les contributeurs se sont également penchés sur la part de l'intime dans la lettre. Politique d'un côté, intime de l'autre: qu'en est-il, alors, de la lettre de particuliers à une administration? Il semble pertinent d'analyser de près ce genre hybride, et quasiment inexploré, qu'est la correspondance que nous désignerons ici par correspondance semi-administrative et qui constitue une variante des lettres de doléance ou de requête telles qu'elles étaient codifiées de longue date dans les manuels épistolaires.

Rien dans la forme de la lettre semi-administrative ne relève en apparence de la correspondance privée. L'écrivant n'écrit ni pour rompre sa solitude, ni pour se confier, mais il s'adresse à un personnage public, choisi précisément pour sa fonction et ses responsabilités officielles, par exemple celles de maire, de président de région ou d'évêque. Son acte vise une efficacité sociale, par exemple l'obtention d'améliorations et l'octroi de soutiens matériels. S'il est avéré que dans le cadre épistolaire l'écrivant ne révèle de lui-même que des éléments partiels, cette autocensure est plus forte encore dans ce contexte. Ainsi l'intime, la vérité d'un être singulier qui se déploierait dans le cadre protégé de la lettre, paraissent-ils destinés à rester à l'arrière-plan. Cependant, c'est de son expérience individuelle que l'épistolier part. Plus encore, elle lui sert de justification, c'est elle qu'il dévoile, déroule. S'il intervient ainsi dans l'espace public, c'est en effet au nom de cette même expérience individuelle qui fait

1. Danièle Poublan, Introduction, in Pierrette Lebrun-Pézerat, Danièle Poublan (dir.), La lettre et le politique: actes du Colloque de Calais, 17-19 septembre 1993, Paris, Honoré Champion, 1996, p. 23. Pour les remarques plus générales sur le genre épistolaire, sur les manuels, sur la sphère intime, nous renvoyons au cadrage théorique figurant dans le numéro 70 des Cahiers d'Études Germaniques. 
office d'argument suffisant. L'écrivant nourrit son récit de ses émotions en même temps que sa lettre permet de se confronter à ses idées, son opinion, sans filtre autre que celui qu'il a lui-même apposé. Dans le même temps, en écrivant à un personnage public, l'épistolier se place sur la scène politique, se positionne vis-à-vis d'elle, signale son droit à la prise de parole et passe ainsi du statut de particulier à celui de citoyen. Le genre de la lettre semi-administrative se situe donc à l'interface entre sphère publique et sphère privée, dans un creuset riche de révélations potentielles.

Dans cette optique, nous proposons dans un premier temps la description formelle de notre corpus, mais aussi de son contenu. Dans un second temps, nous nous interrogerons sur les modalités du repositionnement politique et sur sa signification pour les citoyens allemands au lendemain de 1945. Enfin, en nous fondant sur les lettres de requêtes, nous étudierons les conditions de traitement de l'intime, et ce qu'il révèle sur la société allemande dans l'immédiat de l'après-guerre.

\section{Les lettres de particuliers: description du corpus}

Notre analyse porte sur vingt-deux lettres que des particuliers ont écrites à des autorités allemandes entre le 22 novembre 1945 et le 11 novembre $1948^{2}$. Elles sont envoyées par seize épistoliers différents, comptant parmi eux trois femmes. Il est impossible de déterminer à quelle catégorie socioprofessionnelle ils appartiennent lorsque cela n'est pas précisé, mais les indications dont nous disposons permettent d'affirmer que le panel est très large. La profession est indiquée lorsqu'elle confère un poids à la parole de l'épistolier de par la position sociale qu'il occupe: l'on trouve des fonctionnaires, deux prêtres, un ingénieur en chimie. On peut donc supposer que les origines sociales des autres sept autres épistoliers sont plus modestes. Le langage de certains épistoliers est courant, voire familier. La syntaxe est simplifiée, les phrases sont parfois nominales ou à rallonge. Une seule de ces personnes est originaire d'une ville de taille moyenne - Coblence -, la très faible densité de la zone expliquant que les autres résident dans des petites villes ou villages.

L'âge des épistoliers nous intéresse conformément au " concept de génération ", développé par Aleida Assmann, qui sert à catégoriser les expériences faites par les Allemands durant la guerre ${ }^{3}$. Il nous est difficile de le déterminer, mais de rares indices permettent parfois de l'estimer grossièrement: la mention d'enfants en bas-âge, d'un époux mort à la guerre, d'une situation professionnelle tenant une place élevée dans la hiérarchie sociale. Lorsque les hommes ne mentionnent

2. Ces documents se trouvent dans les fonds suivants: archives étatiques de Fribourg en Br. Dossier C5/1, $\mathrm{N}^{\circ} 928$; archives régionales de Coblence. Dossier 869, $\mathrm{N}^{\circ} 91$; archives régionales de Coblence. Dossier 860, $\mathrm{N}^{\circ} 1111$; archives municipales de Coblence. Dossier 623, $\mathrm{N}^{\circ} 9743$; archives municipales de Coblence. Dossier 623, $\mathrm{N}^{\circ} 8193$.

3. Aleida Assman, Geschichte im Gedächtnis. Von der individuellen Erfahrung zur öffentlichen Inszenierung, München, Beck, 2007, p. 31-69. 
pas de passé militaire ni de détention, il est possible qu'ils aient été trop vieux pour avoir activement pris part à la guerre, ces éléments étant alors source de valorisation dans la société allemande et pouvant appuyer moralement une doléance ${ }^{4}$.

Les épistoliers s'adressent à deux niveaux différents. Leurs lettres sont adressées au gouvernement du Land ou restent cantonnées à l'échelle très locale en étant alors destinées au maire. Sur vingt-deux lettres, dix-sept sont envoyées au gouvernement régional.

Abordant toutes le sujet de la pénurie alimentaire, ces lettres sont des lettres de doléance. Il s'agit d'écrire afin de se plaindre du rationnement ou de formuler une requête précise, mais toutes présentent le point commun de présenter une vision très sombre de la situation. Souvent visiblement rédigées sous l'emprise d'une forte émotion, il n'est pas rare qu'elles abordent différents sujets - dont on ne saisit pas nécessairement d'emblée la concordance. On peut discerner cinq contenus littéraux différents: critique des dysfonctionnements politiques en matière d'alimentation, demande d'une aide financière ou alimentaire, requête en faveur d'une intervention de nature sociale, plainte vis-à-vis de l'occupant ou conseil dispensé aux autorités.

\section{Le repositionnement politique}

Les épistoliers prennent la plume devant une situation jugée intenable et urgente, moins parce qu'ils seraient guidés par leur intérêt immédiat que dans l'intention d'exprimer leur colère. Ce qui peut surprendre est le fait que l'expression de la rancœur est plus fréquente envers les autorités allemandes qu'envers l'occupant. Or, si la littérature secondaire insiste sur la relation entre l'occupant français et les habitants de la zone, ce pendant relationnel n'y est peu ou prou pas évoqué. Il serait faux d'en tirer des conclusions hâtives au vu de la violence des sentiments antifrançais rapportés ailleurs. L'explication est probablement autre: aux uns, les épistoliers imputent des sentiments ancrés dans la tradition et dans la continuité de la guerre - celles d'une haine de l'Allemand vu en ennemi héréditaire - d'où découlerait un comportement cruel - celui de piller les ressources du pays. Aux autres, l'on reproche les modalités de leur action politique. Des sentiments des occupants, on ne pourrait rien changer puisqu'ils relèveraient de l'irrationnel. A contrario, l'on pourrait pallier les lacunes des autorités allemandes en les informant. Ainsi, les reproches se modalisent et s'adaptent dans la conscience des contraintes de l'occupation.

Les reproches ne concernent pas tant l'état de fait que la réaction de l'administration allemande vis-à-vis de la pénurie: quatre épistoliers pensent que les autorités allemandes ne mesurent pas l'ampleur de la situation, et quatre autres qu'elles ne font pas tout ce qui est en leur pouvoir afin d'y remédier.

4. Rolf-Dieter Müller, Gerd. R. Ueberschär, Kriegsende 1945. Die Zerstörung des Deutschen Reiches, Frankfurt a.M., Fischer, 1994, p. 141. 
Cette impression d'un certain laxisme est confirmée, pour trois épistoliers, par la différence entre les rations annoncées dans la presse et les quantités de nourriture effectivement distribuées. Deux, enfin, estiment que l'occupant est la cause de tous les maux, mais attribuent aux autorités allemandes la responsabilité de ne pas savoir s'imposer et affurmer les intérêts allemands.

La population, ayant un rapport plus direct avec les maires et comités de cercle, ne comprend pas les limites de leur action et remet en cause leurs compétences de gestion. Sans surprise, ce sont les distributions insuffisantes qui retiennent principalement l'attention. Un habitant de Müllheim, Robert Wäldin, fait la description suivante:

\begin{abstract}
Wir in Müllheim halten es auch für untragbar, dass man in der Lebensmittelversorgung einen Unterschied macht zwischen Städte [sic] über und unter 20000 Einwohnern. Wir haben hier erst [document illisible] Kal Kartoffeln erhalten und zwar vor ca 10 Wochen und 10 Pfund prof [sic] Kopf, seiter [sic] nichts mehr. Vergangene Woche - wohl als Ersatz - $1 \mathrm{Kg}$. Erbsen und 200 gr Maismehl. In dem Monat gab es [sic] einziges Mal Fett und zwar 50 gr und heute haben wir den 29. September! Vor acht Tage [sic] kriegten wir den Juni-Zucker auf Julikarten mit je 200 gr. [document illisible] und Eier überhaupt keine. Das sind Zuteilungen, die nicht zum Leben, wohl aber zum Sterben reichen ${ }^{5}$."
\end{abstract}

Nous savons que, lorsqu'il prend la plume en février 1947, la pénurie était généralisée. Comme il le suppose, les légumineuses font bien office d'ersatz. L'occupant français en importe afin d'éviter la famine, la farine de maïs étant destinée à remplacer la farine de blé, qui ne réapparaîtra véritablement dans le quotidien qu'en 1948. C'est cette réalité que Wäldin retranscrit par le prisme de son expérience individuelle. La vivacité de son émotion est perceptible à travers ses indications soulignées à la main ainsi qu'à travers la désorganisation syntaxique de son texte. Christian Plantin appelle ce phénomène " les réorganisations de la forme considérée comme basique de l'énoncé » et écrit à ce sujet : « [...] ces formes se présentent comme arrachées au locuteur, involontaires, non pas signifiées, mais vécues, [...] [et] peuvent jouer le rôle rhétorique de garant de sincérité du locuteur ${ }^{6}$." Figurant aussi dans notre corpus, un autre auteur, anonyme, est moins précis, mais, plus révolté, répète quatre fois la quantité totale des pommes de terre reçues dans son village, c'est-à-dire " jusque là, 65 livres, et pas une de plus ». Disposant de moins de moyens langagiers que Robert Wäldin, il convoque ce chiffre, " 65 livres de pommes de terre ", chaque fois qu'il a besoin d'exprimer son indignation :

Die Gemeinde Otterbach besteht aus 90\% Arbeitern. Wir erhielten an Kartoffelzuteilung bis jetzt insgesamt $65 \mathrm{Pfd}$. Kartoffeln u. kein Stück mehr. Wenn Leute, die wirklich vor dem ,Verhungern' stehen, bei dem Bürgermeisteramt nachfragen, von was sie weiter leben sollen, dann bekommen sie von dorten die sehr undemokratische Antwort: ,Wir können euch auch nicht helfen: Kann ein Mensch den ganzen Winter über von 65 Pfd. Kartoffeln leben??? [...] Es ist doch wirklich ein Hohen 65 Pfd. Kartoffeln. Unzählige Familien haben nichts zu essen. [...] Über den Kommunismus wird soviel geschrieben, aber was ist Demokratie in Rheinland-

5. Lettre de Robert Wäldin au président Wohleb datée du 29 février 1947. Archives étatiques de Fribourg. Dossier C5/1, N 928.

6. Christian Plantin, Les bonnes raisons des émotions. Principes et méthode pour l'étude du discours émotionné, Berne, Peter Lang, 2001, p. 138. 
Pfalz??? Bis jetzt 65. Pfd. Kartoffeln, die verzehrt sind, und keine Aussicht, weitere zu bekommen; in diesem Satz ist alles gesagt ${ }^{7}$.

Si la peur ne se dit pas directement, la violence des sentiments se manifeste ici dans le style polémique et l'accumulation de questions rhétoriques.

$\mathrm{Au}$ demeurant, les données factuelles que les historiens ont reconstituées prouvent que ces reproches que les particuliers adressent à leurs élites témoignent de leur méconnaissance des contraintes qui pesaient sur les autorités allemandes : hégémonie et poids réel de l'occupant français dans les processus administratifs et décisionnels allemands, nécessités économiques, logistiques et matérielles. Mais ils sont une précieuse source d'informations quant aux perceptions des habitants de la zone.

Les pistes d'analyse proposées par Daniel Fabre sur la démarche à l'origine de l'écriture " quotidienne " permettent de développer plus avant notre réflexion. D. Fabre écrit, dans Seize terrains d'écriture: "Plus l'écriture est officielle et plus elle requiert une autorité particulière que beaucoup pensent ne pas détenir ${ }^{8}$." La première forme de positionnement politique consiste donc à se sentir légitime dans la prise de parole. Le repositionnement politique réside ici dans la critique. La démarche qui consiste à informer les élites implique inévitablement la formulation d'un avis quant à cette situation. Cela signifie que l'écrivant a conscience de son droit à s'exprimer, l'utilise, et considère également son apport au débat légitime. Cela veut dire également qu'il ne craint pas d'éventuelles conséquences de sa prise de parole et a ainsi bien intégré la différence avec le Troisième Reich où, au cours des derniers mois de la guerre, toute représentation négative des circonstances pouvait être taxée de défaitisme et entraîner des conséquences fatales ${ }^{9}$. Les particuliers exigent d'être pris au sérieux, respectés par les gouvernants. Dans ce contexte, les effets d'annonce - qu'ils jugeaient mensongers - de la presse quant aux distributions de nourriture sont vécus comme un affront, et certains réagissent aux articles de journaux, se réservant un droit de réponse.

Il s'agit donc, pour les épistoliers, de se repositionner face à l'espace public, de s'attribuer un rôle dans la société. Cela va encore plus loin lorsqu'ils prennent la plume au nom d'un groupe dont ils s'érigent en porte-parole. Cette volonté est notamment manifeste chez un autre de nos seize épistoliers, le chimiste Blankenhorn, qui la réitère plusieurs fois au nom de sa ville de Pfullendorf.

Si autocensure il y a, elle n'empêche pas de tenir des propos très directs. Un prêtre de Überlingen s'estime en droit de faire appel au sens du devoir de

7. Lettre anonyme envoyée de Otterbach au Ministre-président Altmeier datée du 5 janvier 1948. Archives régionales de Coblence. Dossier 860, $\mathrm{N}^{\circ}$ 1111. Retranscrite par Peter Brommer, Quellen zur Geschichte von Rheinland-Pfalz während der französischen Besatzung. März 1945 bis August 1949, Mainz, 1985, p. 548.

8. Daniel Fabre, Introduction, Seize terrains d'écriture, in Daniel Fabre (éd.), Par écrit. Ethnologie des écritures quotidiennes, Paris, Éditions de la Maison des sciences de l'homme, 1997, p. 11.

9. À propos de l'accroissement des peines judiciaires auxquelles étaient condamnées les personnes soupçonnées de défaitisme, voir Rudolph Herzog, Heil Hitler, das Schwein ist tot! Lachen unter Hitler - Komik und Humor im Dritten Reich, München, Heyne, 2008. 
tout homme politique. C'est pourquoi il s'adresse directement au président de sa région, le 27 avril 1948, jugeant que certaines administrations allemandes ne font pas leur travail:

\begin{abstract}
dass von gewissen untergeordneten deutschen Dienststellen durchaus nicht immer mit der Festigkeit und dem Verantwortungsbewußtsein, die eine solche Existenzfrage doch auf jeden Fall erheischen, die Interessen der Bevölkerung vertreten werden ${ }^{10}$.
\end{abstract}

La méfiance envers les autorités locales se fait donc le corollaire d'une autre attitude: le passage à une instance supérieure, dernier recours une fois les autres rouages testés en vain. Afin de comprendre les mécanismes en jeu lorsque l'on s'adresse à la plus haute instance de pouvoir, nous nous appuyons sur le travail d'Annie Vénard-Savatosky. D’après elle, cette démarche correspond à « un premier fantasme de démocratie directe ". ${ }^{11}$ La lettre au président intervient parfois au terme d'une série d'autres lettres à des instances politiques intermédiaires, comme c'est le cas pour Hugo Blankenhorn. Chez les épistoliers, cette relation fantasmée donne lieu à une représentation imaginaire des détenteurs du pouvoir: la défiance envers les uns se transforme en une confiance envers l'autre. Il est supposé capable d'améliorer les choses et, surtout, il ignorerait, de par sa position, la réalité de la situation. Aux yeux de certains simples particuliers, informer ce dernier devient un devoir civique.

L'un des arguments de nos épistoliers consiste à se référer au concept de démocratie. Or, avec le recul, il est tentant de relier cet argument à la politique de rééducation des alliés, qui consistait à mettre en place une démocratisation progressive du pays; le concept de démocratie était de ce fait largement évoqué dans la presse et vanté par les voix officielles. Néanmoins, ce n'est pas dans ce sens qu'il est convoqué, à l'époque, par les particuliers. Quand le mot " démocratie " figure dans leurs lettres, il semblerait qu'il s'agisse pour eux d'employer, en référence au fond discursif, un argument supplémentaire dont la signification n'est en réalité pas très bien comprise. En effet, la convocation de ce mot clé est motivée par le but rhétorique mais différentes significations lui sont attribuées. Ainsi, face à la réalité de la disette, l'auteur anonyme qui a été cité plus haut remet-il en question la pertinence d'un régime démocratique en faisant référence au discours anticommuniste déjà en vogue à l'époque ${ }^{12}$. Une autre épistolière, une mère de famille du nom de Hannah Müller, n'attribue pas au concept de signification particulière mais pense que la démocratie doit être accompagnée de moyens financiers ou matériels ${ }^{13}$. Peut-être plus éclairée que les autres en matière de politique, elle qui se dit antifasciste, elle semble partager l’inquiétude des responsables politiques quant aux conséquences de la pénurie

10. Lettre au président Wohleb datée du 27 avril 1948. Archives étatiques de Fribourg. Dossier C5/1, $\mathrm{N}^{\circ} 928$.

11. Annie Vénard-Savatosky, "Lettres au président de la République », in Lebrun-Peyzerat, Poublan, La lettre et le politique, p. 72.

12. Lettre anonyme du 5 janvier 1948 [note 8].

13. Lettre de Hannah Müller à Leo Wohleb daté du 17 janvier 1947. Archives étatiques de Fribourg. Dossier C5/1, N 928. 
sur l'éveil des consciences. Sa démarche demeure néanmoins guidée par l'intérêt privé puisqu'elle cherche à protéger ses enfants. Se concevant victime d'une double injustice - les opposants au nazisme, dont elle fit partie, subissent le même sort que le reste de la population, et ses anciens adversaires ne sont pas moins bien, sinon mieux, lotis qu'elle -, elle mêle également au concept de démocratie son amertume personnelle. Robert Wäldin a une autre position encore: en pleine période cruciale de fondation des structures démocratiques du pays ${ }^{14}$, il estime que le gouvernement aurait mieux à faire que de perdre son temps en tergiversations inutiles. À supposer que Robert Wäldin connaisse le but de ces " réunions ", il n'en mentionne même pas l'objet, et met sur le même plan l'activité législative et la restructuration démocratique du pays.

Ces exemples montrent les limites de la lettre semi-administrative en tant qu'action politique. Il y a certes une prise de parole, un positionnement citoyen du particulier, attitude très significative après les douze ans de terreur national-socialiste. Mais le geste est guidé, sinon par l'intérêt privé, du moins par une perspective individuelle, voire individualiste. Cette démarche est à replacer dans le contexte, plus général, de l'éloignement voire de l'indifférence de beaucoup d'Allemands vis-à-vis de la politique au lendemain de la seconde guerre mondiale. C'est pourquoi il est pertinent d'analyser aussi l'autre versant des lettres semi|administratives, la part de l'intime, qu'il soit placé au second plan, comme dans les lettres de doléance, ou au premier, comme pour les lettres de requête.

\section{Les conditions d'émergence de l'intime: un intime amputé et stéréotypé}

Les épistoliers tracent d'eux un autoportrait qui trahit en réalité bien plus leurs angoisses qu'il ne définit leurs conditions de vie. L'autoportrait permet donc ici d'établir la liste des maux de l'époque à savoir, par ordre d'évocation : la pénurie de nourriture, l'inquiétude pour les proches, l'inquiétude vis-à-vis de l'arrivée de l'hiver, les difficultés à se livrer au troc, les conditions de logement, et enfin le manque de biens matériels ou d'argent.

Le froid hivernal aggrave la menace sur l'intégrité physique. Le manque de combustibles est rendu particulièrement insupportable par la vie dans les logements précaires, les particuliers ayant vu leurs habitations touchées par les bombes ou complètement détruites. Rappelons que la destruction partielle des habitations peut s'avérer aussi gênante que leur destruction totale. Dans les deux cas, les gens sont exposés aux variations climatiques et les organismes sont malmenés. L'absence de logement décent entraînait également d'autres complications auxquelles on ne pense pas d'emblée, mais qui sont évoquées par les épistoliers. Qui a perdu sa maison a également perdu ses biens, ce

14. Les thèmes abordés alors concernent notamment la constitution d'un gouvernement provisoire, le programme du gouvernement badois et la future rédaction de la constitution allemande. 
qui complique considérablement la survie. Quatre des épistoliers y voient un problème bien spécifique: ils ne possèdent plus de biens à échanger au marché noir ou à troquer contre de la nourriture. Ces descriptions rendent donc bien compte de la spirale de la misère. Lors de la description des conditions de vie, il est également beaucoup fait mention des conditions familiales. L'état de santé des enfants est alors pour les parents le motif déclencheur de l'acte d'écrire. Ils sont décrits à travers les critères du sexe mais surtout de l'âge, ce qui permet de souligner leur fragilité. Le nombre d'enfants est également immédiatement mentionné, puisque chaque bouche de plus à nourrir signifie des diffıcultés démultipliées pour les familles. Apparaît ici un véritable topos rhétorique. Les épistoliers débutent souvent leur lettre en évoquant ou même en présentant leur famille, raison d'écriture en même temps qu'argument principal. L'impossibilité de les nourrir est ensuite immédiatement évoquée. Les rations qui parviennent jusqu'aux consommateurs sont insuffisantes alors que les réserves de la famille sont épuisées: on retrouve encore une fois cette impression d'impasse, de situation insurmontable, qui provoque le désespoir en même temps que l'acte d'écrire.

D'autres facteurs sont mentionnés. Femmes enceintes, fraîchement accouchées ou nouveau-nés sont des variations possibles venant renforcer le topos des conditions familiales. Un épistolier, qui signe de l'initiale M., évoque sa femme enceinte tout à la fin de la lettre, comme s'il se souvenait au dernier moment d'un argument supplémentaire pouvant jouer en sa faveur. Cette mention se fait à juste titre, étant donné les menaces particulières qui pèsent sur cette catégorie de population. Autre variation possible du tableau de la misère privée, le célibat des femmes, alors livrées à elles-mêmes et chargées de pourvoir seules aux besoins de leurs enfants. Ainsi, du mari de Hannah Müller, nous savons qu'il a "sacrifié sa vie à l'état nazi, et certainement pas de son plein gré ${ }^{15}$ ", ce qui signifie vraisemblablement qu'il est tombé au front en tant que soldat de la Wehrmacht.

Au-delà de la peinture des circonstances privées se dessinent donc des catégories de la misère. Ces éléments sont récurrents dans les documents d'époque. Ils sont des sujets de préoccupations pour les particuliers, mais aussi pour les autorités ou les associations caritatives. De ce fait, ils acquièrent vite le statut de topoï dans le paysage discursif, dans un premier temps conformément à leur pouvoir rhétorique, via l'émotion provoquée. Il serait malvenu et inapproprié de tracer une ligne franche qui distinguerait la rhétorique de ce qui serait alors des émotions véritables ${ }^{16}$. Néanmoins, ces catégories sont également utilisées pour

15. " [...] mein Mann, der sein Leben gewiss nicht freiwillig dem Nazi-Staat geopfert hat [...]. "

16. Dans une lettre à sa femme, datée du 25 novembre 1946, dans laquelle il décrit une distribution de biens, Joel Carl Welty traduit bien ce paradoxe apparent entre réelle nécessité, mise en scène de la misère et émotion. " Es stimmt traurig, den Geschichten von Kummer und Leid zuzuhören und Tränen fließen zu sehen, wenn Menschen in den Hilfsausschuß kommen und mit leiser, befangener Stimme um Schuhe für ihre Kinder, um Lebensmittel oder Kleidung fragen. Oft bringen sie die Kleinen mit und demonstrieren ihre Not ». Joel Carl Welty, Das Hungerjahr in 
caractériser l'époque, avec tous les enjeux de l'exploitation de la victimisation de la société allemande au lendemain de la seconde guerre mondiale.

Qui réunit certaines de ces catégories peut prétendre au statut de victime, et donc espérer une quelconque compensation, qu'elle soit matérielle, financière ou morale, sous la forme de la compassion. Pour les épistoliers qui formulent une requête, l'enjeu consiste donc à être caractérisés en tant que victimes. Certains s'y refusent, ou du moins s'en défendent. D'autres posent clairement ce statut avant de formuler une requête. Ainsi, avant de solliciter une intervention en faveur de son fils, emprisonné, selon elle, de façon injuste par l'occupant, Mme Malchus se présente et énumère toutes les difficultés auxquelles elle fait face. Elle les mentionne toutes soigneusement, de son veuvage et ses jeunes enfants, arguments les plus marquants qui arrivent en tête, à l'arrivée redoutée $\mathrm{du}$ froid. Toutes ces raisons sont bien entendu un argument en faveur de la libération de son fils, puisqu'elle affirme, dans sa détresse, avoir besoin de lui. Mais elles ne contribuent pas avant tout à étayer son statut de victime. La typographie des arguments, rédigés les uns en-dessous des autres, confirme cette impression d'exposé. La lettre commence par les mots suivants:

Geehrter Herr Oberbürgermeister! Im Vertrauen wende ich mich an Sie, Herr Oberbürgermeister, mit der herzlichen Bitte, mir in einer schmerzlichen Angelegenheit zu helfen. Ich bin kriegshinterbliebene Witwe mit 5 minderjährigen Kindern. Ich bin bombengeschädigt und total ausgeplündert, sodaß ich sehr notdürftig und sparsam leben muß. Meine AngestelltenRente beträgt nur monatl.76.10 Rm. Mein Junge, erster Ehe, Namens Karlheinz Schmidt, Schreinerlehrling bei der Fa. Voilh. Kühn, Hohenzollernstr. ist seit dem 30. Juli als [?] von den Franzosen verhaftet worden und sitzt seit dieser Zeit im Gefängnis der Karthause. Der Junge ist meine einzige Stütze, er ist sehr fleißig und ordentlich und half unsere Familie ernähren und baute selbst unser Häuschen wieder auf und muß noch vor Winter vieles instand setzen. Jetzt fehlt mir das Kind. Mein Junge und ich kommen nicht darüber hinweg, daß er unschuldig für die ganze Firma Kühn leiden soll. [...].

À ces caractéristiques s'ajoute, le cas échéant, le statut d'opposant au nazisme, comme le fait Hannah Müller. Ces mentions s'inscrivent pleinement dans la réflexion sur la tendance ou l'attitude de victimisation adoptée par les Allemands au lendemain de l'Armistice.

La stéréotypisation passe également par ce qui n'est pas dit. Au récit des faits rapportés manque tout ce qui pourrait désavouer l'épistolier, jeter une ombre sur son irréprochabilité, sur son identité de victime à part entière. Il revient au chercheur de compléter le tableau. Les activités sur les marchés parallèles - marché noir, Hamstern - ne sont évoquées que lorsqu'elles ne peuvent avoir lieu. Cela revient à concéder à demi-mot que l'on s'y adonnerait le cas échant. Pourtant, aucun épistolier n'avoue ouvertement s'y livrer, comme c'était pourtant le cas de la majorité de la population. ${ }^{17}$ Dans la même veine, toutes les conséquences de la faim ne sont pas décrites. L'impact physiologique et

der Französischen Zone des geteilten Deutschland, 1946-1947. Traduit de l'anglais par Christine Ternes, Koblenz, Fuck, 1995, p. 64.

17. C'est le cas dans toute l'Allemagne. À titre comparatif, Vincent Bignong et Isabelle Bilon-Hoefkens considèrent que les échanges illégaux représentent, dès janvier 1946, environ 50 \% des échanges dans la zone d'occupation américaine. Vincent Bignon, Isabelle Bilon-Hoefkens, " L'impact de la 
psychologique est abordé, mais pas les "fourvoiements " auxquels la misère mène: mendicité, vols, prostitution alimentaire. Or il y a bel et bien eu une véritable " criminalité alimentaire " dans l'immédiat de l'après-guerre, au développement si considérable qu'elle comptait également parmi les sources de subsistance. ${ }^{18}$ Ce genre de comportements est tout à fait susceptible de concerner les auteurs de nos lettres, puisque les premiers à s'y résoudre sont précisément ceux dont les provisions sont épuisées et qui n'ont plus les moyens de s'adonner au Hamstern ou au marché noir. Dans la même veine, si les épistoliers évoquent leurs conditions familiales, ils n'en mentionnent que des aspects partiels. S'ils insistent sur la vulnérabilité des femmes seules ou enfants, une grande partie de la réalité des relations familiales est occultée. La faim se répercute sur les relations au sein de la cellule familiale. Au-delà des tensions créées par les conditions de vie dans les ruines, la promiscuité, la diffıcile réinsertion des pères de famille dans la structure familiale, ou encore la nouvelle indépendance des femmes, le manque de calories pèse, lui aussi, sur les familles. Les épistoliers ne nous livrent donc qu'un intime amputé, destiné à l'extérieur et donc poli et partiel.

\section{Conclusion}

Ainsi, la lettre semi-administrative s'avère offrir elle aussi une porte vers l'intimité de l'individu, et constitue une approche pour l'historien de la vie quotidienne. Néanmoins le destinataire est toujours présent de manière implicite. Aux limites du propre regard sur soi s'ajoute le regard que l'on veut présenter à l'autre. Au récit des faits rapportés manque ainsi tout ce qui pourrait désavouer l'écrivant, jeter une ombre sur son irréprochabilité et donc nuire à son message. Mais le genre semi-administratif, de par sa bipolarité, permet un changement de perspective. L'on détourne ainsi le regard de la vérité intime de l'individu pour explorer sa relation au pouvoir, ainsi que, plus généralement, les conditions de la démocratie dans la société. Cette double approche se révèle riche d'enseignements. Dans le cas de la lettre semi-administrative en Allemagne au lendemain de la Seconde Guerre, elle permet d'observer dans un premier temps la réappropriation de la citoyenneté au sein de la population civile, après les années de dictature nazie. Mais au-delà de ce premier constat, le genre témoigne aussi des limites de la redémocratisation instaurée par les alliés. Enfin, l'étude de la peinture de l'intime amputé et stéréotypé, sitôt qu'elle ne se fait pas à travers un seul cas en particulier mais plutôt à travers un kaléidoscope, nous permet d'observer l'émergence d'un paysage discursif commun. Dans le cas de l'Allemagne, la question d'une victimisation s'est posée dans le cadre de l'occupation, donc dès les années 1945-1949. Replacées dans un contexte plus

réforme monétaire de juin 1948 sur les prix au marché noir en Bavière ", Francia, tome 40, n 1 , janvier-mars 2008, p. 126. Voir à ce propos les comptes rendus politiques, op. cit.

18. Karl-Heinz Rothenberger, Die Hungerjahre nach dem Zweiten Weltkrieg. Ernährungs- und Landwirtschaft in Rheinland-Pfalz 1945-1950, Boppard am Rhein, Boldt, 1980, p. 135. 
large - discours politique, presse ${ }^{19}$ - ces lettres de doléance viennent enrichir le débat sur la victimisation de la population allemande et son rapport au passé nazi après la guerre.

19. Voir notamment notre thèse de doctorat, Marjorie Maquet, Typologie et enjeux des discours sur la misère dans la zone d'occupation française en Allemagne entre 1945 et 1950, soutenue à l'université Toulouse Jean-Jaurès le 4 décembre 2015, p. 281-302, p. 343-353. 
